\title{
Multi-proteomic Analysis Revealed Distinct Protein Profiles in Cerebrospinal Fluid of Patients Between Anti-NMDAR Encephalitis NORSE and Cryptogenic NORSE
}

\section{Dongmei Wang}

Southern Medical University Nanfang Hospital

\section{Yongming Wu}

Southern Medical University Nanfang Hospital

\section{Yue Pan}

Southern Medical University Nanfang Hospital

\section{Shengnan Wang}

Southern Medical University Nanfang Hospital

\section{Guanghui Liu}

Southern Medical University Nanfang Hospital

\section{Yibo Gao}

Chinese Academy of Medical Sciences \& Peking Union Medical College

Kaibiao Xu ( $\square$ xukaibiao@163.com)

Nanfang Hospital, Southern Medical University https://orcid.org/0000-0001-8278-6438

\section{Research}

Keywords: New-onset refractory status epilepticus, cerebrospinal fluid proteome, anti-NMDAR encephalitis, cryptogenic-NORSE, humoral immune, cellular immune, neurodegeneration

Posted Date: November 2nd, 2021

DOI: https://doi.org/10.21203/rs.3.rs-1029260/v1

License: (c) (1) This work is licensed under a Creative Commons Attribution 4.0 International License. Read Full License 


\section{Abstract}

Background: New-onset refractory status epilepticus (NORSE) caused by anti-N-methyl-d-aspartate receptor (anti-NMDAR) encephalitis and cryptogenic etiologies are various in clinical features. The underlying mechanisms of the diseases have still remained elusive.

Methods: 6 patients with anti-NMDAR encephalitis NORSE, 5 with cryptogenic NORSE (C-NORSE), and 5 controls were enrolled. Clinical data, including clinical features, cerebrospinal fluid (CSF) samples, and cranial images were collected. CSF samples were tested for FAIMS based quantitative proteomic analysis, immunome protein microarray, and inflammatory cytokine array. Bioinformatics and immunostaining were used for interpretation and verification.

Results: The clinical features of anti-NMDAR encephalitis NORSE and C-NORSE patients were similar to those reported previously. Proteomic analysis revealed that 101 proteins (63 up-regulated and 38 downregulated) and 56 proteins (42 up-regulated and 14 down-regulated) changed in CSF samples of antiNMDAR encephalitis NORSE and C-NORSE patients, respectively. The mean fold-change of the upregulated proteins, namely up-proteomic score in this study, was positively associated with the severity of disease, including ICU stay, mRS score at discharge, and time taken for patients awaking from a coma. The distribution of changed proteins in CSF between the two diseases were quite different. Pathways of humoral immune response, wound healing, and epigenetic regulation of transcription were up-regulated in anti-NMDAR encephalitis NORSE. Pathways of innate and lymphocyte mediated immune response, synaptic functions, ubiquitination, and cell apoptosis were up-regulated in C-NORSE group, suggesting that C-NORSE could undergo neurodegeneration. This was consistent with a mouse model of SE, which showed high ubiquitination and cell apoptosis in CA1 to CA3 regions of hippocampus. Immunome microarray analysis demonstrated high autoantibody targeting 48 proteins in CSF samples of antiNMDAR encephalitis NORSE. However, in C-NORSE, the reaction was kept at a very low level. Inflammatory cytokine array, unexpectedly, showed no remarkable changes except for decreased level of interleukin-6 (IL-6) in both anti-NMDAR encephalitis NORSE and C-NORSE.

Conclusions: The present study suggests that the up-proteomic score of CSF could be a promising indicator for assessment of the severity of anti-NMDAR encephalitis NORSE and C-NORSE. The distinct CSF proteomes imply different pathogenic mechanisms of the two diseases, and immunotherapy strategies as well.

\section{Introduction}

Status epilepticus (SE) is the second most frequent and life-threatening neurologic emergency, especially if refractory (RSE) [1]. The annual incidence of RSE is 3.4-7.2 per 100000 individuals, and new-onset RSE (NORSE) represents about $20 \%$ of it [2]. NORSE is characterized by the patient without active epilepsy or other pre-existing relevant neurological disorder, with new-onset of RSE without a clear acute or active structural, toxic or metabolic cause [3]. Most of the patients with NORSE are super refractory and require 
prolonged intensive care unit (ICU) treatment and poor outcome. A mortality rate of $12 \%$ has been seen in children and even higher in adults (16-27\%) [2]. It is insufficient to treat the epilepsy alone without attempting to target the underlying pathogenesis.

NORSE is a very heterogeneous condition owning to various causes. Autoimmune encephalitis ( $A E, 37 \%)$ is the most commonly identified etiology, followed by infection pathogens (8\%) [4]. Anti-N-methyl-daspartate receptor (NMDAR) encephalitis is the single highest frequent cause. However, about half of NORSE cases, after thorough work-up for etiology screening, remain no definite cause determinated. These cases are considered as cryptogenic NORSE (C-NORSE) [5]. Comparing with anti-NMDAR encephalitis, C-NORSE is more frequently associated with prodromal fever, status epilepticus, ventilatory support, symmetric brain MRI abnormalities, and a worse outcome, has less frequent involuntary movements, absent psychobehavioral symptoms, cerebrospinal fluid (CSF) oligoclonal bands, or tumor association [5]. The underlying mechanisms remain unclear and further investigation is warranted.

Given the putative causal role of autoimmune or inflammation in NORSE, early initiation of immunotherapy is recommended [6, 7]. Evidence from case series showed favorable effects on seizure frequency and cognition and even successful treatment by first-line immunotherapy (steroids, immunoglobulins, and plasma exchange) /and second-line immunotherapy (tacrolimus, rituximab, cyclophosphamide, anakinra, etc.) [5, 8-10]. However, the role of immunotherapy is still controversial, because of unclear etiology and pathology [11]. It seems less effective in children [5]. The mechanisms involved in epileptogenesis are broad and heterogeneous, including aberrant neurogenesis, reactive gliosis, mossy fibre sprouting, blood-brain barrier (BBB) dysfunction, and ion channel dysfunction, which lead to abnormal cellular and functional plasticity and imbalance of excitation and inhibition $[12,13]$. These events could be attributed to molecular pathways (BDNF, mTOR, TGF $\beta$, neuroinflammation, oxidative stress, etc.), transcriptomic alterations (ATF3, CREB, JAK-STAT, NRF2, etc.), and epigenetic alterations (microRNA, DNA methylation, histone acetylation) [12]. To elucidate and comparing the underlying mechanisms of anti-NMDAR encephalitis NORSE and C-NORSE, the present retrospective study was conducted via several proteomic approaches, including high-field asymmetric waveform ion mobility spectrometry (FAIMS), immunome protein microarray, and inflammatory cytokine array, to analyze the CSF samples from patients with NORSE. Distinct protein profiles and involved pathways were observed between the two diseases.

\section{Materials And Methods}

\section{Patients' selection}

In the present study, a total of 11 patients with critical NORSE, including 6 anti-NMDAR encephalitis NORSE and 5 C-NORSE, were admitted to the Neurology Department and Neurological Intensive Care Unit (NICU) of Nanfang Hospital (Guangzhou, China) between January 1, 2015 and October 1, 2019, were enrolled. 5 patients with cerebrovascular diseases were used as controls. 
The diagnosis of anti-NMDAR encephalitis was performed according to the following criteria presented by Xu et al.[14] :1) acute onset of 1 or more of the 8 major groups of manifestations: psychosis, memory deficit, speech disturbance, seizures, movement disorder, loss of consciousness, autonomic dysfunction, and central hypoventilation; 2) CSF samples were positive for anti-NMDAR antibodies (cell-based assay); and 3) reasonable exclusion of other disorders.

The diagnosis of C-NORSE was undertaken on the basis of the following criteria: 1) no history of epilepsy or seizures; 2) RSE: SE that persists despite administration of at least two appropriate parenteral medications (e.g., benzodiazepine); 3) unknown etiology after extensive evaluation[15].

\section{Ethical statement, informed consent, and sample collection}

This study was approved by the Ethics Committee of Nanfang Hospital, Southern Medical University (Permit No. NFEC-2021-001). Written informed consent was obtained from all the patients or their family members. Patients' CSF samples were collected from the first lumbar puncture within $24 \mathrm{~h}$ after admission. The residual samples were frozen and stored at $-80^{\circ} \mathrm{C}$ until further analysis.

\section{Clinical data collection}

Demographic and clinical data, including CSF test results, autoimmune antibodies, ovarian teratoma, magnetic resonance imaging (MRI) or computed tomography (CT) findings, length of stay at ICU, duration of ventilation, anti-epileptic drug (AED) regimen, and immunotherapy were collected. The immunotherapy included steroids, plasma exchange (PE), intravenous immunoglobulin (IVIG), and immunosuppressant. All the patients were followed-up in our clinic, and the prognosis was evaluated by modified Rankin scale (mRS) at the time of discharge (short-term prognosis) and at 6 months' follow-up (long-term prognosis). The indications for ICU admission included severe anti-NMDAR encephalitis with ovarian teratoma requiring surgery, $\mathrm{SE}$, the necessity of undergoing mechanical ventilation, and hemodynamic instability.

\section{FAIMS-based quantitative proteomic analysis}

Proteomic analysis of CSF was carried out by PTM Biolabs Inc. (Hangzhou, China). Briefly, separated CSF (with $1 \%$ protease inhibitor cocktail) was centrifuged at $1000 \mathrm{~g}$ for $10 \mathrm{~min}$. The supernatant concentration was determined with a bicinchoninic acid (BCA) assay kit according to the manufacturer's instructions. To perform digestion, equal amount of proteins from each sample was digested with trypsin. Digested peptides were analyzed with liquid chromatography-tandem mass spectrometry (LC-MS/MS) by using an Orbitrap Exploris 480 mass spectrometer (Thermo Fisher Scientific, Waltham, MA, USA) coupled with an Easy-nLC 1200 UPLC system (Thermo Fisher Scientific) and the FAIMS Pro system (Thermo Fisher Scientific). Raw MS data were processed with Proteome Discoverer software (ver. 2.4.1.15; Thermo Fisher Scientific) using the UniProt Homo sapiens database. Proteins (with > 1.5-fold changes) were further analyzed by bioinformatics. 
Gene Ontology (GO) annotations were derived from UniProt-GOA database (http://www.ebi.ac.uk/GOA/). A two-tailed Fisher's exact test was employed to test the enrichment of the significantly changed proteins. The heatmap was generated with quantified LFQ value using MeV 4.9.0 software. The STRING database (ver. 11.0) was utilized to search for all the significantly changed proteins (confidence score $\geq 0.4$ ) for constructing protein-protein interaction (PPI) networks. The PPI networks were visualized by Cytoscape 3.6.1 software (Cytoscape Inc., Boston, MA, USA).

\section{Immunome protein microarray}

Sengenics KREX ${ }^{\mathrm{TM}}$ Immunome Protein Micoarray system was employed for the purpose of highthroughput sequencing of autoantibodies as conducted by Univ-bio Inc. (Shanghai, China). Briefly, CSF samples were diluted (1:10) with SAB buffer. Pipette $4 \mathrm{ml}$ of diluted sample into Quadriperm chamber. Place the prepared slides that were coated with 1622 proteins into the corresponding chamber, and then, incubate at $50 \mathrm{rpm}$ for $2 \mathrm{~h}$ at $20^{\circ} \mathrm{C}$. After thrice washing with $30 \mathrm{ml} \mathrm{SAB}$ buffer, the slides were sunk into staining box containing $200 \mathrm{ml}$ diluted Cy3-antihuman IgG (1:1000), and were further incubated at 50 $\mathrm{rpm}$ for $2 \mathrm{~h}$ at $20^{\circ} \mathrm{C}$. After thrice washing with $200 \mathrm{ml} \mathrm{SAB}$ buffer for $5 \mathrm{~min}$, the slides were subsequently vacuum-dried and scanned by Agilent G2565CA Microarray Scanner System (Agilent Technologies Inc., Santa Clara, CA, USA). The relative fluorescence unit (RFU) of each array spot was extracted by GenePix Pro 7. The data were analyzed according to a standard procedure. The biomarkers were identified using penetrance fold change (PFC)-based method: penetrance frequency (\%) $\geq 10$ and PFC $\geq 2$. Cy3BSA, IgG, intraprotein $\mathrm{CV} \%$, and pooled normal sera QC were used for quality control. The gene-disease association data were collected from the Open Targets platform (http://www.targetvalidation.org/).

\section{Inflammatory cytokine array}

Semi-quantitative measurement of human inflammatory cytokines was performed by using C-Series Human Inflammation Antibody Array 1 Kit (Cat. GSH-INF-1; RayBiotech, Inc., Guangzhou, China). Briefly, $100 \mu \mathrm{l} \mathrm{CSF}$ sample without dilution was analyzed and incubated at $4{ }^{\circ} \mathrm{C}$ overnight. After washing 10 times with $250 \mu \mathrm{l} 1 \times$ Wash Buffer l/well for $10 \mathrm{~s}$, and subsequently, washing 6 times with $250 \mu \mathrm{l} 1 \times$ Wash Buffer II for $10 \mathrm{~s}, 80 \mu \mathrm{l}$ of biotin labeled detection antibody cocktail was added to each well and incubated at room temperature for $2 \mathrm{~h}$. Wash the chip as described above and add $80 \mu \mathrm{l} /$ well Cy3-streptavidin solution and incubate for $1 \mathrm{~h}$ at room temperature in the dark. After washing, the chip was scanned by the InnoScan 300 Microarray Scanner (Innopsys Inc., Paris, France) at $532 \mathrm{~nm}$. The raw data were extracted and processed by GSH-INF-1-SW. The normalized RFU values were further statistically analyzed.

\section{Immunostaining}

A mouse model of SE was established as previously reported. Cryosections of brain of rat or mouse were prepared by using CM1950 cryostat (Leica, Wetzlar, Germany). HEK293T cells were transiently transfected with pcDNA3.1-NMDAR plasmid and subsequently cultured for $24 \mathrm{~h}$. The sections and HEK293T cells were fixed with cold acetone for $10 \mathrm{~min}$. After thrice washing with phosphate-buffered saline with Tween ${ }^{\circledR}$ detergent (PBST), the slides were blocked by using $1 \%$ bovine serum albumin 
(BSA)/PBST at room temperature for $1 \mathrm{~h}$. Discarded the blocking buffer and added CSF (without dilution), anti-ubiquitin antibody (10201-2-AP; dilution, 1:50; Proteintech, Rosemont, IL, USA), anti-PAK1 antibody (21401-1-AP; dilution, 1:50; Proteintech, Rosemont, IL, USA) or anti-SYN1 antibody (5297; dilution, 1:200; CST, Danvers, MA, USA) and incubated at room temperature for $2 \mathrm{~h}$ or at $4{ }^{\circ} \mathrm{C}$ overnight . It was then attempted to thrice wash with PBST for 5 min. Added goat anti-human/rabbit IgG DyLight ${ }^{\circledR} 594$ secondary antibody (ab96909/ab96885; dilution, 1:200; Abcam, Cambridge, UK), and incubated at room temperature for $1 \mathrm{~h}$. After washing, the slides were sealed and the images were taken by using IX73 microscope (Olympus, Tokyo, Japan).

\section{Terminal deoxynucleotidyl transferase dUTP nick end labeling (TUNEL) assay}

TUNEL assay was carried out by using a KGA7061 kit (KeyGEN BioTECH, Nanjing, China) according to the manufacturer's instructions. Briefly, the fixed cryosections of brain of mouse were incubated with terminal deoxynucleotidyl transferase (TdT) reaction solution supplemented with biotin-11-dUTP at $37{ }^{\circ} \mathrm{C}$ for $60 \mathrm{~min}$. After thrice washing with PBS for $5 \mathrm{~min}$, a solution of TRITC-labeled streptavidin was added and incubated at $37^{\circ} \mathrm{C}$ for $30 \mathrm{~min}$. The slides were washed as mentioned above, and the images were captured by using the IX73 microscope (Olympus, Tokyo, Japan).

\section{Statistical analysis}

The inflammatory cytokine array data were statistically analyzed by using one-way analysis of variance (ANOVA). Data were presented as mean \pm standard deviation (SD). Correlation of a protein's fold-change with time requiring for patients to awake from coma was statistically analyzed by using the Spearman's or Pearson's correlation analysis ( $95 \%$ confidence interval). A P-value $<0.05$ was considered statistically significant. Data were processed and plotted via Prism 8 software (GraphPad Software Inc., San Diego, CA, USA).

\section{Results}

\section{Clinical characteristic}

In the current research, 6 patients with anti-NMDAR encephalitis NORSE (R1-R6), 5 with C-NORSE (E1-E5), and 5 controls (C1-C5) were enrolled. The grouping and clinical features of the patients are presented in Table 1.

The severity, outcomes and relapses of patients with anti-NMDAR encephalitis are correlated with the anti-NDMAR antibody level of CSF $[16,17]$. To elucidate different stages of anti-NMDAR encephalitis NORSE, patients without history of immunotherapy $(R 4, R 5)$ and refractory to IVIG (R6, without clinical improvement or antibody reduction), and those with history of immunotherapy and antibody reduction (R2, R3) or negative conversion (R1) were included (Fig. S1). All the anti-NMDAR encephalitis NORSE patients received AEDs. Among all the C-NORSE patients, no definite pathogenic factor was confirmed. 
Patients with definite vascular diseases (e.g., cerebral infarction, hemorrhage, cerebral venous sinus thrombosis, cerebral arteriovenous fistula, and vascular malformation) were selected as controls.

The cranial images and electroencephalogram (EEG) findings of patients are illustrated in Figs. S2-S3. For patients with anti-NMDAR encephalitis NORSE, EEG revealed diffused delta waves with increased amplitude. CT/MRI of brain was unremarkable or with leukoaraiosis. The MRI findings in the C-NROSE were not specific with scattered abnormal signals in the cortex or subcortex. Besides, a number of patients were found with complete normal MRI findings.

\section{Proteomic up-regulation index in CSF was correlated with the severity of patients with anti-NMDAR encephalitis NORSE and C-NORSE}

To determine the underlying mechanisms of anti-NMDAR encephalitis NORSE and C-NORSE and whether C-NORSE is also an immune-mediated inflammatory disease, FAIMS-based quantitative proteomic analysis of CSF samples was conducted (Fig. 1A). 12239 unique peptides, 1797 proteins, and 1675 quantifiable proteins were identified (Fig. 1B, Additional file 1). The peptides were distributed between 720 amino acids, which were coincident with trypsin digestion. In nearly $30 \%$ of proteins, sequence coverage was above $20 \%$. Proteins with fold-change over 1.5 and P-value less than 0.05 were considered as significantly changed. 63 and 38 proteins were up- and down-regulated in CSF samples in patients with anti-NMDAR encephalitis NORSE, respectively (Fig.1B, Additional file 2); 42 and 14 proteins were upand down-regulated in CSF samples in patients with C-NORSE, respectively (Fig. 1B, Additional file 3).

Putting proteomes and clinical characteristics together, we found that up-regulated proteins were positively associated with the length of stay in ICU and mRS score at discharge. These two factors reflected severity of disease and neurological function of the patients. Compared to patients of R1, R2, and R3, proteins were more significantly up-regulated in R4, R5, and R6, accompanying with longer duration in ICU and higher mRS score at discharge. The same pattern was also observed between E1 and E2 versus E3, E4 and E5 (Fig. 2A). In addition, the mean fold-change of all up- or downregulated proteins was calculated as the proteomic score. Since some patients' ICU stay period were not quite accurate because of pass away or discharge ahead, time needed to awake for patients in a coma was used as another indicator of disease severity. Interestingly, the proteomic score of up-regulated proteins showed approximately the same trend with the time from sample collection to awoke (Fig. 2B-C). To narrow-down the highly correlated proteins, Spearman's or Pearson's correlation analysis was undertaken for each changed proteins. 7 proteins in anti-NMDAR encephalitis NORSE group, IGLV3-27, IGKV1-39, LI6R, SECTM1, ICAM1, GRN, and CD82, were found. These proteins' mean fold change or named as refined proteomic score was highly logarithmic correlated with time needed to awake $\left(\mathrm{R}^{2}=0.9739\right.$, Fig. S4). In C-NORSE group, 9 proteins were found, including HIST2H2AC, PPP3CC, PDXP, SLC9A6, HDHD1, CPLX2, SH3BGRL, PSMA3, and CAPZB (Fig. S5). There were no significant differences in the profile of down-regulated proteins and the down-proteomic scores among the patients within antiNMDAR encephalitis NORSE group, and within C-NORSE group as well (Fig. 2A, 2B). 
The distribution of changed proteins in CSF samples was quite different between two groups. Venny analysis showed barely overlap of up- or down-regulated proteins (Fig. 2D). Subcellular localization of the changed proteins revealed that in anti-NMDAR encephalitis NORSE group, about $57 \%$ were extracellular proteins, while in C-NORSE group, $29 \%, 25 \%$, and $18 \%$ were cytoplasmic, extracellular, and nuclear proteins, respectively (Fig. S6). Principal component analysis confirmed this discrepancy. There was no obvious clustering among all the samples (Fig. S7), while samples with high up-proteomic score were clustered well into different groups according to the type of disease (Fig. S8). These data imply that anti-NMDAR encephalitis NORSE and C-NORSE could underlie different mechanisms, leading to SE.

\section{Proteins involved in humoral immune response, epigenetic regulation, and wound healing changed in CSF samples of anti-NMDAR encephalitis NORSE}

GO enrichment analysis of changed proteins in anti-NMDAR encephalitis group showed that up-regulated proteins were highly enriched in humoral immune response and epigenetic regulation of gene expression, including immunoglobulins, HLA-A, HLA-B, F5, APOE, PCSK9, CD74, GRN, HIST1H4A, HIST2H2AC, H3F3A, ENPP2, GANAB, GALNT1, PLD4, PLAUR, ERAP2, PRDX4, PLOD1, etc. (Fig. 3A). Down-regulated proteins were involved in negative regulation of coagulation and wound healing, such as CFH, MGP, FGA, S100A8, FGB, MAPK1, AHSG, EPB42, SERPINE1, CTSG, HRG, SPTAN1, FGG, KNG1, LTF, CKB, APOA2, ZC4H2,GP1BA, PLCB3, FBN1, CTGF, DDAH1, B3GAT1, DNER, PLS3, GLUL, etc. (Fig. 3A). The majority of these proteins were estimated in a network (Fig. 3B). We further analyzed top three samples with high upproteomic score, R4, R5 and R6, and more changed proteins were observed (Fig. S9, S10). However, the pathways mentioned above were highly enriched as well (Fig. S11).

As expected, high level of immunoglobulin appeared in CSF samples of patients with anti-NMDAR encephalitis NORSE (Fig. 1B, 2A, S1). To determine the immuno-reactivity and antibody profile of CSF, immunome protein microarray assay, which consists of more than 1600 proteins, was performed. R3, R4, R5, and R6 showed a high reaction, and 48 targeted biomarkers were identified (Fig. 3C, Additional file 46), demonstrating a comprehensive immune response in anti-NMDAR encephalitis NORSE patients. To indicate the inflammatory response level, cytokine protein microarray was also carried out. It was noted that 10 cytokines, including interferon- $\gamma$ (IFN- $\gamma$ ), interleukin (IL)-1a, IL-1ß, IL-10, IL-13, IL-4, IL-6, IL-8, monocyte chemotactic protein-1 (MCP-1), and tumor necrosis factor-a (TNF-a) were analyzed. Unexpectedly, none of them were significantly up-regulated either in anti-NMDAR encephalitis NORSE group or C-NORSE group (Fig. 3D, Additional file 7). Besides, IL-6, an important proinflammatory cytokine, was down-regulated, though its receptor IL-6R was increased in R3, R4, R5, E4, and E6.

\section{CSF proteomes of C-NORSE indicated a neurodegeneration process}

GO enrichment analysis of up-regulated proteins of C-NORSE group showed that pathways of regulation of innate immune response and lymphocyte mediated immunity were enriched, such as SCL9A6, HLA-B, UBQLN1, SYN1, PAK1, FCER2, LEP, PLAUR, STAT1, PSMA3, and complements C4A and C4B (Fig. 4A, upper panel). The reduced proteins were enriched in Fc receptor signaling pathway and immune response 
mediated by immunoglobulin (Fig. 4A, lower panel). This was consistent with the low reactivity in immunome protein microarray analysis (Fig. 3C, Fig. S12).

In addition to immune response, axon extension, dendrite, and synaptic-involved biological processes were highly enriched (Fig. 4A). 41 of 42 up-regulated proteins were related to synapse, especially presynapse (Fig. 4B). Moreover, 36 of these proteins were related to ubiquitination (Fig. 4C); 29 formed several networks or interactions (Fig. 4D). Furthermore, cell apoptosis, phagocytosis, peptidase, and proteasome pathways were highly enriched (Fig. 4A). STAT cascade, which is important for regulation of cell apoptosis, appeared. In samples with top three up-proteomic scores, E3 , E4, and E5 (Fig. S13), cell apoptosis-associated biological processes could be observed (Fig. S14). The above-mentioned findings imply that C-NORSE might undergo a neurodegeneration process. To verify this hypothesis, a mouse model of SE was established. Surprisingly, two synapse proteins detected, PAK1 and SYN1, were increased consistently with FAIMS (Fig. S15) and an obvious ubiquitination and cell apoptosis could be observed in the stratum pyramidale of CA1 to CA3 regions of hippocampus (Fig. 5).

\section{Discussion}

In the present study, we retrospectively collected clinical data and CSF samples of 6 anti-NMDAR encephalitis NORSE patients, 5 C-NORSE patients, and 5 controls with definite diagnosis of vascular diseases. We compared the clinical profiles and CSF proteomes of the patients. We found that the CSF protein profiles of anti-NMDAR encephalitis NORSE and C-NORSE were significantly changed compared to the controls. The disease severity of anti-NMDAR encephalitis NORSE and C-NORSE patients was associated with the fold-change of up-regulated proteins or up-proteomic score. Further analyses implied that humoral immune response in anti-NMDAR encephalitis NORSE as expected, while cellular immune response and neuronal degeneration in C-NORSE. More studies are still warranted.

The clinical comparisons between patients with C-NORSE and anti-NMDAR encephalitis NORSE were scarcely conducted. [5] [5] [5] [5] [5] In the current study, we found that imaging findings were consistent with those of a previous research in terms of cryptogenic NORSE, and MRI of brain showed symmetric hyperintensities on T2/fluid-attenuated inversion recovery (FLAIR) MRI of brain[18]. In patients with antiNMDAR encephalitis, the cranial images were not specific with scattered hyperintensities on FLAIR sequences $^{[14]}$. The patients also showed mild abnormal signals in MRI/CT findings, which is consistent with those achievements reported previously $[14,18]$.

Recently, proteomics has been widely used to elucidate the disease mechanisms. However, rare research has concentrated on anti-NMDAR encephalitis NORSE and C-NORSE. Based on the proteomic analysis of the CSF samples, we show that anti-NMDAR encephalitis NORSE and C-NORSE are completely different diseases although both possess similar clinical features, such as seizures, changed mental status, and response to immunotherapy. As barely overlap of the up- or down-regulated proteins was demonstrated, the underlying mechanisms of the diseases could be quite different. These data suggest that antiNMDAR encephalitis NORSE and C-NORSE are distinguishable by CSF protein profile within a molecular 
level, and the up-regulated proteins could be used as indicators of progress of diseases. However, further profound researches should be conducted to elucidate the inner connections.

Based on the results of our research, we found that the antibodies and proteins in anti-NMDAR encephalitis NORSE patients were increased dramatically compared to those in the controls. These changes revealed the immune mechanisms in anti-NMDAR encephalitis NORSE patients. These results might explain the effectiveness of immunotherapy, including steroids and immunosuppressant drugs.

The mechanism of immunotherapy for C-NORSE has still remained elusive. A study demonstrated that plasma exchange was effective for severe cases [9]. Other researches showed that patients may benefit from other types of immunotherapy, such as steroids, IVIG, and immunosuppressant drugs $[5,8]$. The exact role of the immunotherapy has remained obscure. The present study showed that antibody production in the C-NORSE group was not obvious compared with that in the control group. Hence, we suggest that patients may benefit more from IVIG and plasma exchange that may regulate and modulate or even enhance the immune system, rather than administration of steroids or immunosuppressant drugs that may suppress the immune system. This result should be interpreted with caution, since this was a small observational study with limited sample size, and larger clinical trials should be conducted to confirm the mentioned hypothesis.

The inflammatory biomarkers of CSF revealed that only IL-6 level in was reduced. This phenomenon was different from the literature that the levels of inflammatory biomarkers in anti-NMDAR encephalitis and SE patients were significantly elevated $[19,20]$. The involvement of inflammatory cytokines in the pathogenesis of SE has been declared. Animal studies have shown that the levels of IL-1b, IL-6, and TNFa were enhanced in glial cells [21]. The deviation of this study presented may be related to the disease stage, timing of sampling or immunotherapy some patients received before admission to our center. The severity of inflammation may be influenced by the immunotherapy, which was consistent with our findings, in which the levels of inflammatory cytokines were significantly decreased in the follow-up period compared with those in acute stage [22]. In addition, the proteins involved in negative regulation of wound healing pathways decreased in anti-NMDAR encephalitis NORSE which might contribute the reduction of inflammatory cytokines.

We also attempted to elucidate the mechanisms of anti-NMDAR encephalitis NORSE and C-NORSE (Fig. 6). GO enrichment analysis of differentially expressed proteins of anti-NMDAR encephalitis showed that up-regulated proteins were enriched in epigenetic regulation of gene expression and humoral immune response, while down-regulated ones were enriched in negative regulation of coagulation and wound healing. These results indicated that humoral immunity and wound healing could play important roles (mutual restriction) in the pathogenesis of anti-NMDAR encephalitis NORSE.

In C-NORSE patients, 41 of the 42 proteins were related to the synaptic or presynaptic functions, and the majority of them were involved in innate and lymphocyte-mediated immune response, ubiquitination, and cell apoptosis. Coincidently, the AEs of anti-intracellular neuronal antigens are neurodegeneration disorders mediated by $T$ cell cytotoxicity and usually have less response to immunotherapy than anti- 
membranal antigen AEs such as anti-NMDAR encephalitis [23]. Patient E5, though negative for screened known $A E$ antibodies, its CSF immunostaining on rat brain sections showed both cytoplasmic and membranal immunoreaction which made $E 5$ a suspected AE. These suggest that cellular immune response and neuronal damage might play a substantial role in the pathogenesis of C-NORSE.

The present study demonstrated the possible underlying mechanisms of anti-NMDAR encephalitis NORSE and C-NORSE. The results broadened our understanding on the immune status of the diseases and the proper therapeutic strategy for the patients. Besides, there are a number of shortcomings, including being a single-center observational research, small sample size, and the necessity of conducting further researches to confirm the results.

\section{Conclusions}

In conclusion, the protein profiles of anti-NMDAR encephalitis NORSE and C-NORSE significantly differed. The underlying mechanisms of the diseases were quite different that may provide different treatments for the patents. However, more research is warranted to elucidate the molecular mechanisms of the diseases.

\section{Declarations}

\section{SUPPLEMENTARY INFROMATION}

The supplementary Figure S1-S15 are submitted within Figure file for initial submission. The supplementary tables are submitted as following Additional files.

\section{Acknowledgements}

We would like to thank enrolled patients for their participation in the study. We also thank the colleagues of our Neurology Department and Laboratory of Diagnosis of Neurological Disorder and Neuro-protection Research for their advice and support through the course of this work.

\section{Authors' Contributions}

DW, YG, KX are responsible for concepts and design. YP, KX, YW and SW are responsible for data collecting and statistical analysis. GL and YP are responsible for immunostaining tests of all the patients. All authors acquired, analyzed, and interpreted the data. All authors contributed intellectually. The manuscript was prepared by DW and KX. All authors reviewed and made critical revisions to the manuscript.

\section{Funding}

This work was supported by National Natural Science Foundation of China (82171602 to Xu, 82071484 to Wu), President Foundation of Nanfang Hospital (2019B007 to Wang, 2020B008 to Xu), 
Medical Science and Technology Foundation of Guangdong Province (A2021151 to Xu) and Natural Science Foundation of Guangdong Province (2019A1515011760 to Wu).

\section{Availability of data and materials}

The datasets used and/or analysed during the current study are available from the corresponding author on reasonable request.

\section{Ethics approval and consent to participate}

This study was approved by the Ethics Committee of Nanfang Hospital, Southern Medical University (Permit No. NFEC-2021-001).

\section{Consent for publication}

Written informed consent was obtained from all the patients or their family members.

\section{Competing interests}

The authors declare no competing or financial interests.

\section{Author details}

${ }^{1}$ Department of Neurology, Nanfang Hospital, Southern Medical University, Guangzhou 510515, China;

${ }^{2}$ Department of Thoracic Surgery, National Cancer Center/National Clinical Research Center for Cancer/Cancer Hospital, Chinese Academy of Medical Sciences and Peking Union Medical College, Beijing 100021, China; ${ }^{3}$ State Key Laboratory of Molecular Oncology, National Cancer Center/National Clinical Research Center for Cancer/Cancer Hospital, Chinese Academy of Medical Sciences and Peking Union Medical College, Beijing 100021, China.

\section{References}

1. Vezzani A, Dingledine R, Rossetti AO: Immunity and inflammation in status epilepticus and its sequelae: possibilities for therapeutic application. Expert Review of Neurotherapeutics 2015, 15:1081-1092.

2. Mantoan Ritter L, Nashef L: New-onset refractory status epilepticus (NORSE). Practical Neurology 2021, 21:119-127.

3. Hirsch LJ, Gaspard N, van Baalen A, Nabbout R, Demeret S, Loddenkemper T, Navarro V, Specchio N, Lagae L, Rossetti AO, et al: Proposed consensus definitions for new-onset refractory status epilepticus (NORSE), febrile infection-related epilepsy syndrome (FIRES), and related conditions. Epilepsia 2018, 59:739-744.

4. Gaspard N, Foreman BP, Alvarez V, Kang CC, Probasco JC, Jongeling AC, Meyers E, Espinera A, Haas KF, Schmitt SE, et al: New-onset refractory status epilepticus: Etiology, clinical features, and outcome. 
Neurology 2015, 85:1604-1613.

5. lizuka T, Kanazawa N, Kaneko J, Tominaga N, Nonoda Y, Hara A, Onozawa Y, Asari H, Hata T, Kaneko J, et al: Cryptogenic NORSE: Its distinctive clinical features and response to immunotherapy. Neurol Neuroimmunol Neuroinflamm 2017, 4:e396.

6. Specchio N, Pietrafusa N: New-onset refractory status epilepticus and febrile infection-related epilepsy syndrome. Developmental Medicine \& Child Neurology 2020, 62:897-905.

7. Jang Y, Kim DW, Yang KI, Byun J-I, Seo J-G, No YJ, Kang KW, Kim D, Kim KT, Cho YW, Lee S-T: Clinical Approach to Autoimmune Epilepsy. Journal of Clinical Neurology 2020, 16:519.

8. Gall CRE, Jumma O, Mohanraj R: Five cases of new onset refractory status epilepticus (NORSE) syndrome: Outcomes with early immunotherapy. Seizure 2013, 22:217-220.

9. Li J, Saldivar C, Maganti RK: Plasma exchange in cryptogenic new onset refractory status epilepticus. Seizure 2013, 22:70-73.

10. Kodama S, Arai N, Hagiwara A, Kimura A, Takeuchi S: A favorable outcome of intensive immunotherapies for new-onset refractory status epilepticus (NORSE). Journal of Intensive Care $2018,6$.

11. Gaspard N, Hirsch LJ, Sculier C, Loddenkemper T, van Baalen A, Lancrenon J, Emmery M, Specchio N, Farias-Moeller R, Wong N, Nabbout R: New-onset refractory status epilepticus (NORSE) and febrile infection-related epilepsy syndrome (FIRES): State of the art and perspectives. Epilepsia 2018, 59:745-752.

12. Devinsky O, Vezzani A, O'Brien TJ, Jette N, Scheffer IE, de Curtis M, Perucca P: Epilepsy. Nature Reviews Disease Primers 2018, 4.

13. Staley K: Molecular mechanisms of epilepsy. Nature Neuroscience 2015, 18:367-372.

14. Xu X, Lu Q, Huang Y, Fan S, Zhou L, Yuan J, Yang X, Ren H, Sun D, Dai Y, et al: Anti-NMDAR encephalitis: A single-center, longitudinal study in China. Neurol Neuroimmunol Neuroinflamm 2020, 7.

15. Wang D, Pan Y, Huang K, Lin Z, Xie Z, Liu G, Wu Y, Wang S: Is rat hippocampus section immunostaining an indicator for immunotherapy in cryptogenic adult new-onset refractory status epilepticus (NORSE)? Seizure 2020, 76:131-136.

16. Dalmau J, Lancaster E, Martinez-Hernandez E, Rosenfeld MR, Balice-Gordon R: Clinical experience and laboratory investigations in patients with anti-NMDAR encephalitis. Lancet Neurol 2011, 10:6374.

17. Gresa-Arribas N, Titulaer MJ, Torrents A, Aguilar E, McCracken L, Leypoldt F, Gleichman AJ, BaliceGordon R, Rosenfeld MR, Lynch D, et al: Antibody titres at diagnosis and during follow-up of antiNMDA receptor encephalitis: a retrospective study. The Lancet Neurology 2014, 13:167-177.

18. Cartagena AM, Young GB, Lee DH, Mirsattari SM: Reversible and irreversible cranial MRI findings associated with status epilepticus. Epilepsy Behav 2014, 33:24-30. 
19. Liu CY, Zhu J, Zheng XY, Ma C, Wang X: Anti-N-Methyl-D-aspartate Receptor Encephalitis: A Severe, Potentially Reversible Autoimmune Encephalitis. Mediators Inflamm 2017, 2017:6361479.

20. Terrone G, Frigerio F, Balosso S, Ravizza T, Vezzani A: Inflammation and reactive oxygen species in status epilepticus: Biomarkers and implications for therapy. Epilepsy \& Behavior 2019, 101:106275.

21. Simoni MGD, Perego C, Ravizza T, Moneta D, Conti M, Marchesi F, Luigi AD, Garattini S, Vezzani A: Inflammatory cytokines and related genes are induced in the rat hippocampus by limbic status epilepticus. European Journal of Neuroscience 2000, 12:2623-2633.

22. Zou C, Pei S, Yan W, Lu Q, Zhong X, Chen Q, Pan S, Wang Z, Wang H, Zheng D: Cerebrospinal Fluid Osteopontin and Inflammation-Associated Cytokines in Patients With Anti-N-Methyl-D-Aspartate Receptor Encephalitis. Frontiers in Neurology 2020, 11.

23. Bien CG, Vincent A, Barnett MH, Becker AJ, Blumcke I, Graus F, Jellinger KA, Reuss DE, Ribalta T, Schlegel J, et al: Immunopathology of autoantibody-associated encephalitides: clues for pathogenesis. Brain 2012, 135:1622-1638.

\section{Figures}


A

\section{Sample aquisition \Sample preparation > LC-MS/MS > Data analysis}
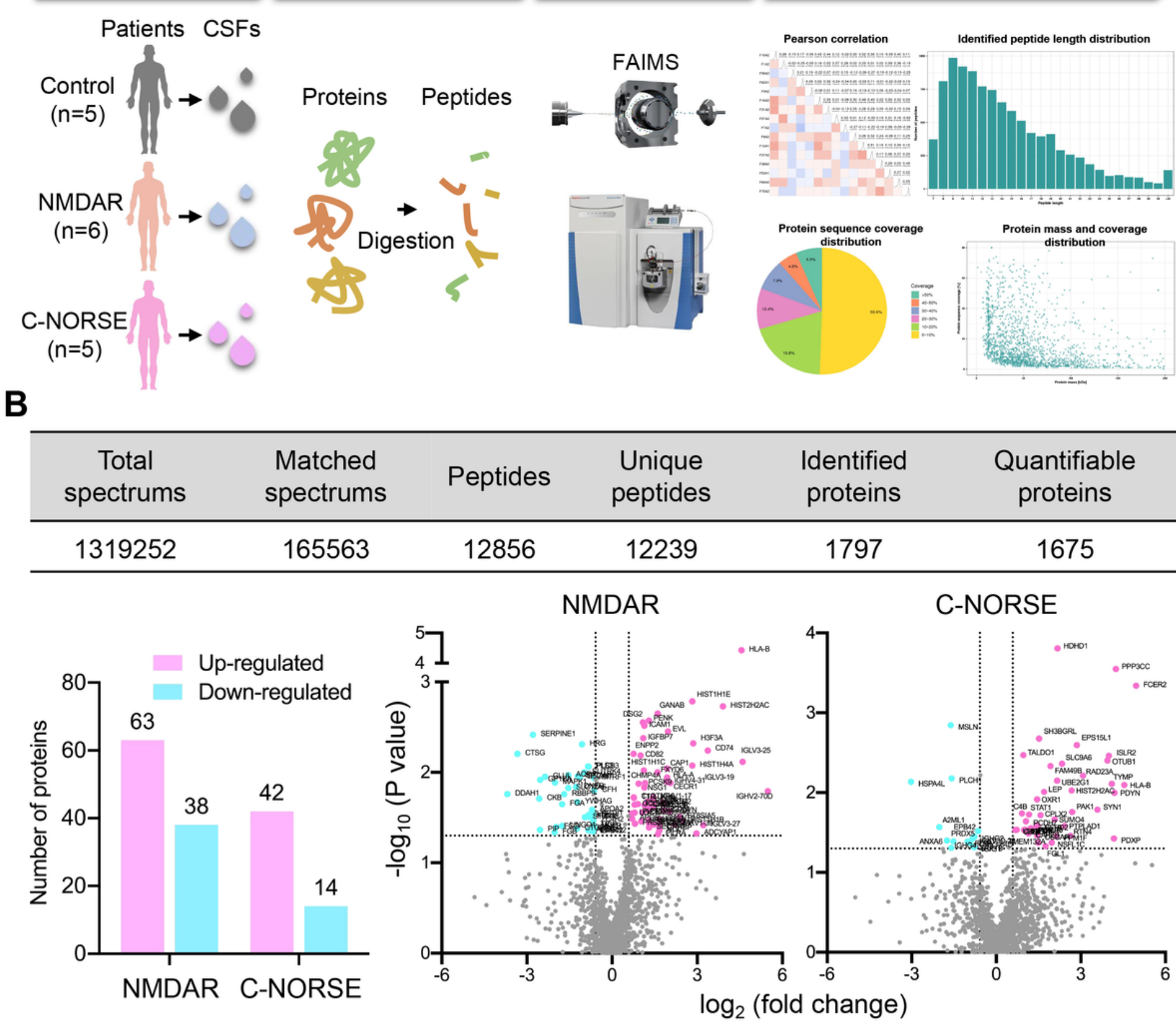

\section{Figure 1}

Quantitative proteomic analysis of CSF samples from anti-NMDAR encephalitis NORSE and C-NORSE patients. (A) Workflow of quantitative proteomic analysis. CSF samples of the patients were digested and applied to LC-MS/MS (FAIMS) for quantification. Identified peptides were mainly distributed between 7-20 amino acids that was coincident with trypsin digestion. In $70 \%$ of proteins, sequence coverage was under 20\%. (B) Number of identified mass spectra, peptides, and proteins are illustrated. 12239 unique peptides and 1797 proteins were identified, and 1675 proteins were quantifiable. Proteins with fold-change over 1.5 and P-value less than 0.05 were considered as significantly changed. 63 and 38 proteins were up- and 
down-regulated in CSF samples of anti-NMDAR encephalitis NORSE patients, respectively. 42 and 14 proteins were up- and down-regulated in CSF samples of C-NORSE patients, respectively (column chart). The volcano plots showed proteins that were significantly changed (up-regulated in pink and downregulated in light blue). CSF, cerebrospinal fluid; FAIMS, high-field asymmetric waveform ion mobility spectrometry.

A

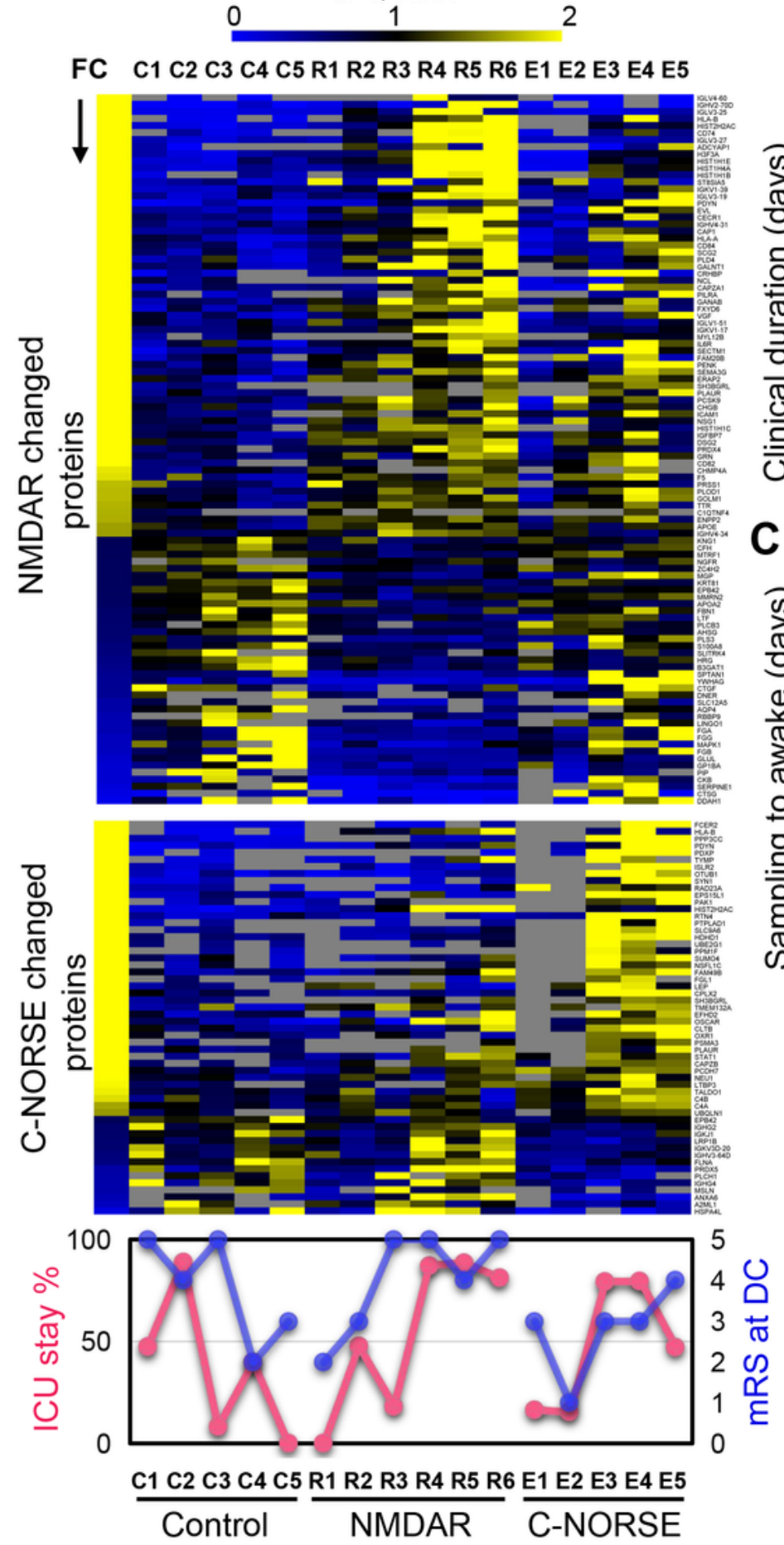

B
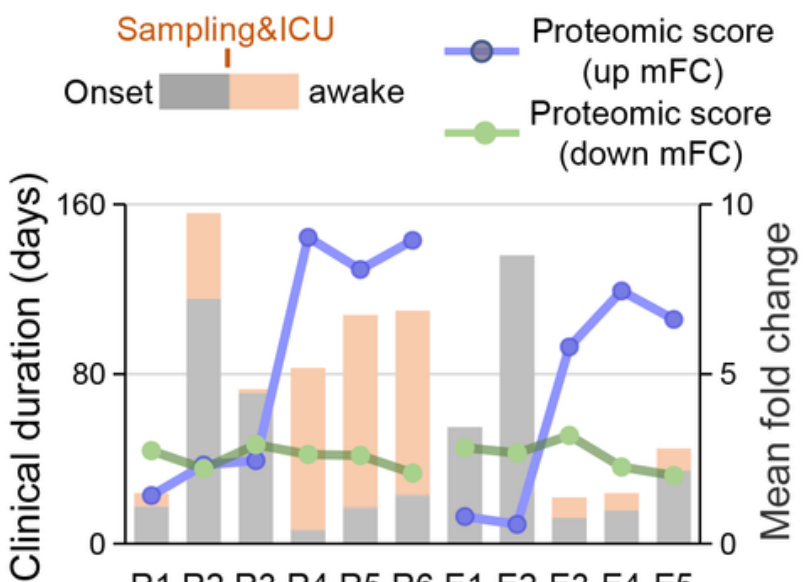

R1 R2 R3 R4 R5 R6 E1 E2 E3 E4 E5

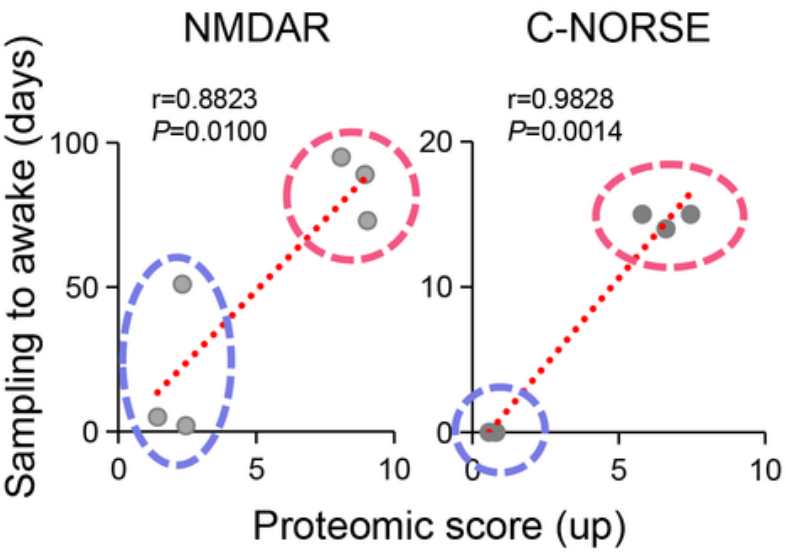

D

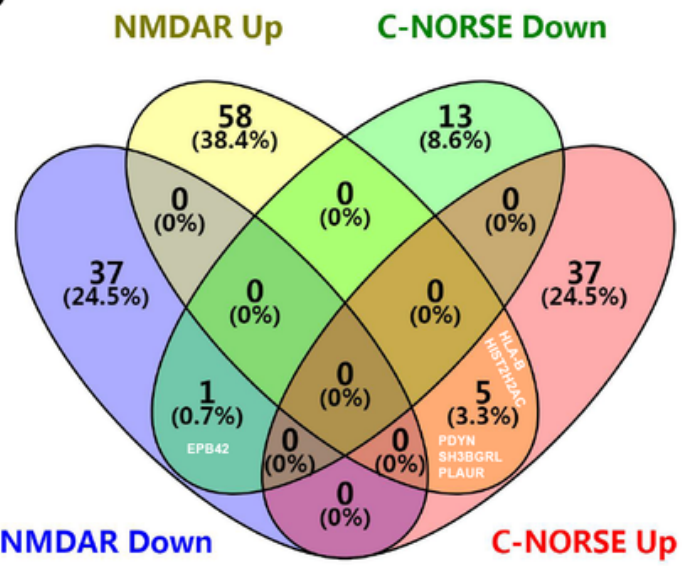

Figure 2 
Proteome changes in CSF samples of anti-NMDAR encephalitis NORSE and C-NORSE patients. (A) Heat map of LFQ values of significantly changed proteins in CSF samples of anti-NMDAR encephalitis NORSE (upper panel) and C-NORSE (middle panel) patients. Unavailable LFQ values were shown in gray. Proteins were sorted by fold-change. From disease onset to the end of hospitalization at ICU in our center was considered as whole clinical duration. Proportion of ICU hospitalization was calculated (ICU stay\% = length of stay at ICU/whole clinical duration, red line, left $Y$ axis). mRS score (blue line, right $Y$ axis) of the patients was assessed with a standard procedure, while ICU discharge (lower panel). (B) Clinical process and proteomic score. The columns (left $Y$ axis) showed time from disease onset to ICU admission (gray) and time from sampling to awake for patients in a coma (brown). CSF samples were collected at the day of admission to ICU. Mean fold-change of all up- or down-regulated proteins was named as proteomic score (right $Y$ axis). (C) Pearson's correlation analysis between awake time needed and proteomic score. (D) Venny analysis of all 157 changed proteins between NMDAR and C-NORSE. 1 protein, EPB42, was down-regulated in both NMDAR and C-NORSE. 5 proteins, HLA-B, HIST2H2AC, PDYN, SH3BGRL, and PLAUR were up-regulated in both NMDAR and C-NORSE groups. LFQ, label-free quantification; FC, foldchange; $\mathrm{mFC}$, mean fold-change; ICU, intensive care unit; DC, discharge. 
A

GO enrichment (BP) of up-regulated proteins

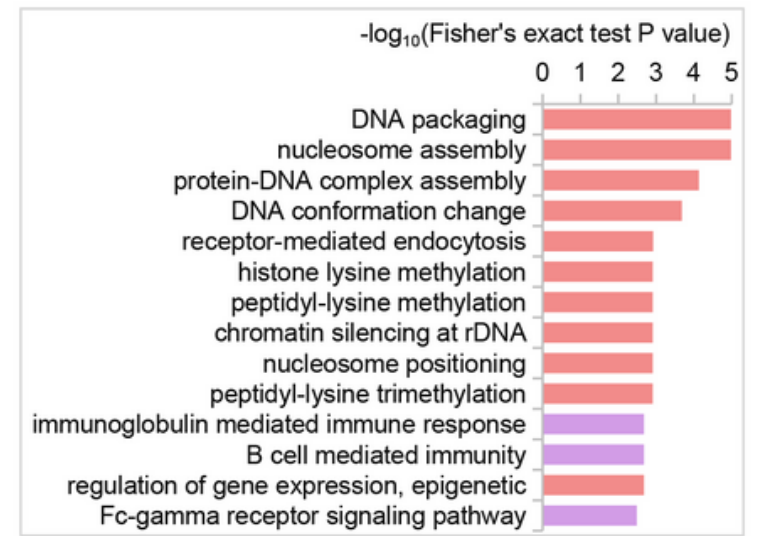

B

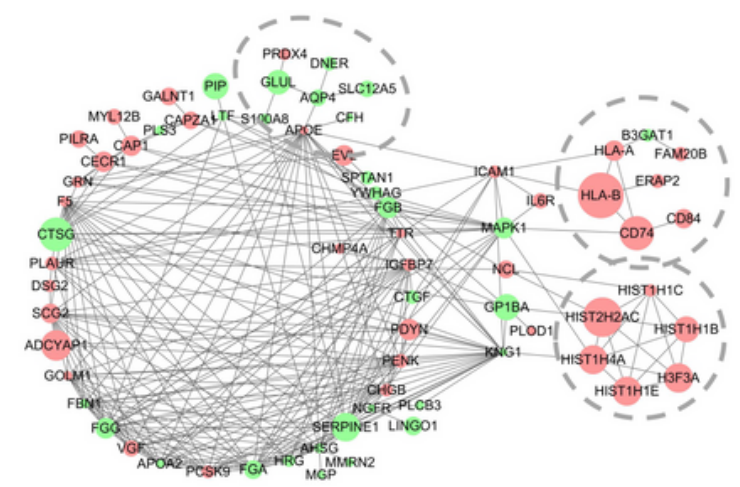

GO enrichment (BP) of down-regulated proteins

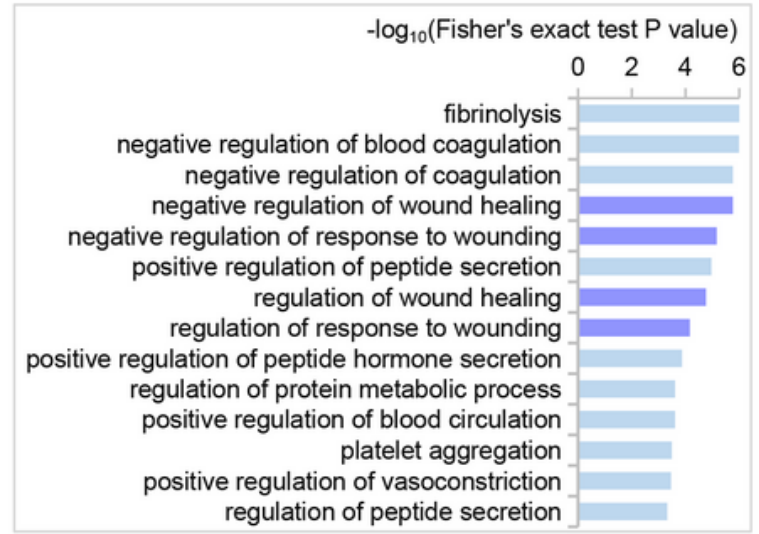

C

auto-immunome

$1.0 \quad$ IFC $\quad 4.0$

C1 C2 C3 C4 C5 R1 R2 R3 R4 R5 R6 E1 E2 E3 E4 E5

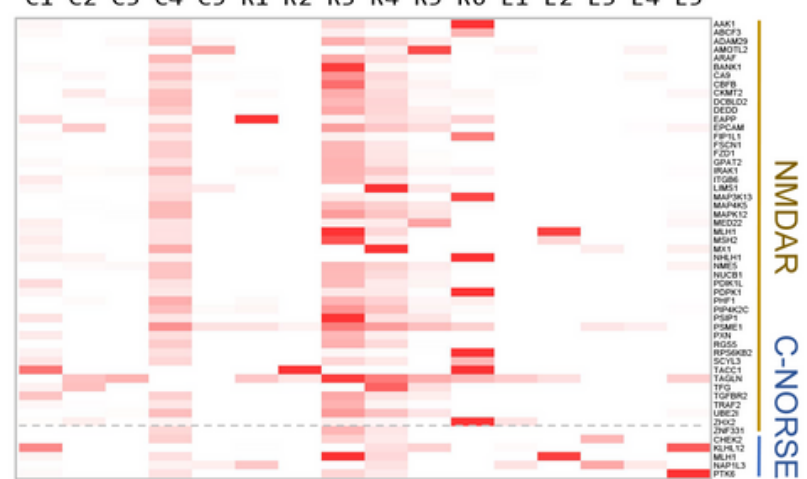

D
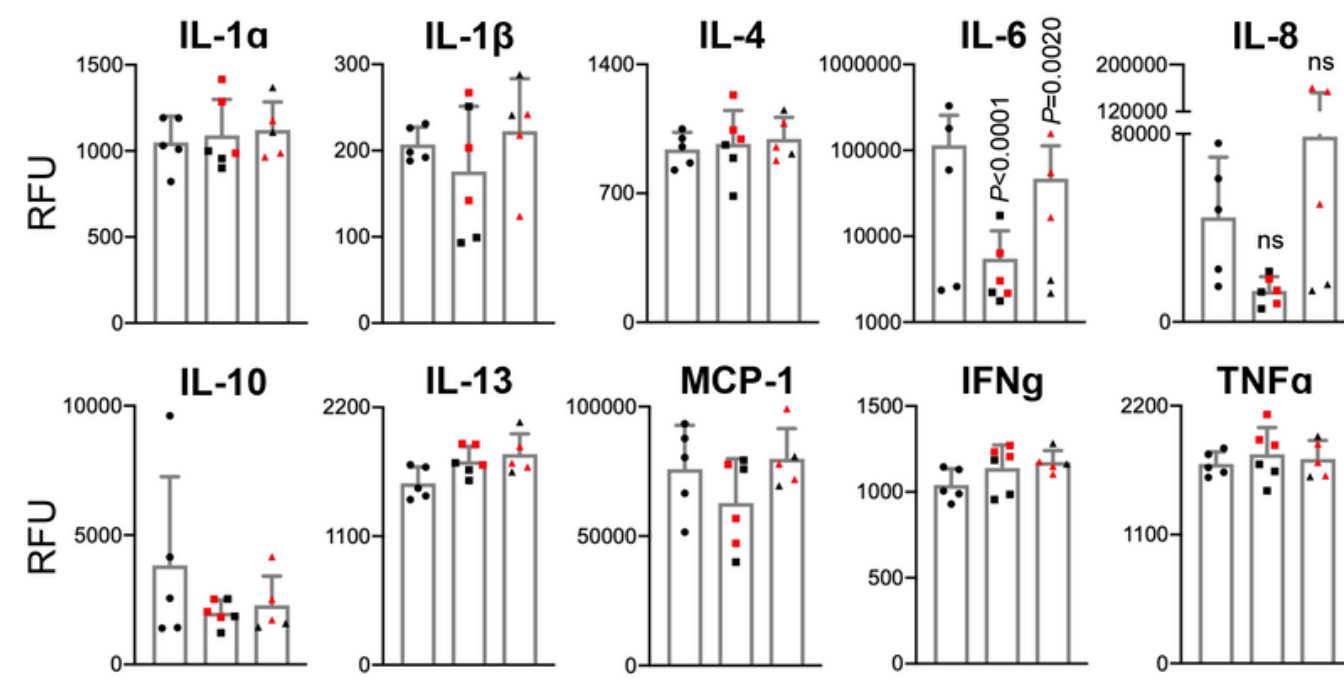

NMDAR

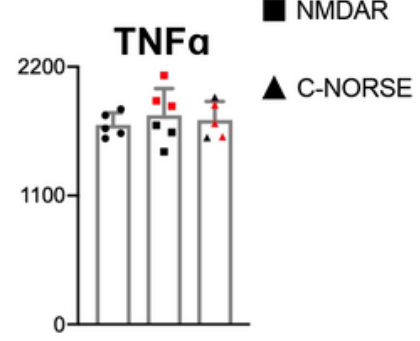

\section{Figure 3}

Proteins involved in humoral immune, wound healing, and epigenetic regulation changed in CSF samples of anti-NMDAR encephalitis NORSE patients. (A) GO (biological processes) enrichment analysis of up(left panel) and down (right panel)-regulated proteins in NMDAR group. The enrichment of the proteins was assessed by the Fisher's exact test. (B) Protein-protein interaction (PPI) network analysis of changed proteins. The PPI networks were constructed on STRING database and reconstructed by Cytoscape 
software. Red, up-regulated proteins; green, down-regulated proteins; bubble size represents relative foldchange. (C) Immunome protein microarray assay of CSF samples from patients. Heat map demonstrated 48 biomarkers in NMDAR and 5 biomarkers in C-NORSE, which were identified from penetance foldchange analysis. (D) The relative level of inflammatory cytokine in CSF samples of anti-NMDAR encephalitis and C-NORSE patients. Red dots represent top 3 patients of high up-proteomic score. BP, biological processes; GO, gene ontology; IFC, individual fold-change; IFN- $\gamma$, including interferon- $\gamma$; IL-, interleukin; MCP-1, monocyte chemotactic protein-1; ns, non-significant; RFU, relative fluorescence unit; TNF-a, tumor necrosis factor-a. 
A

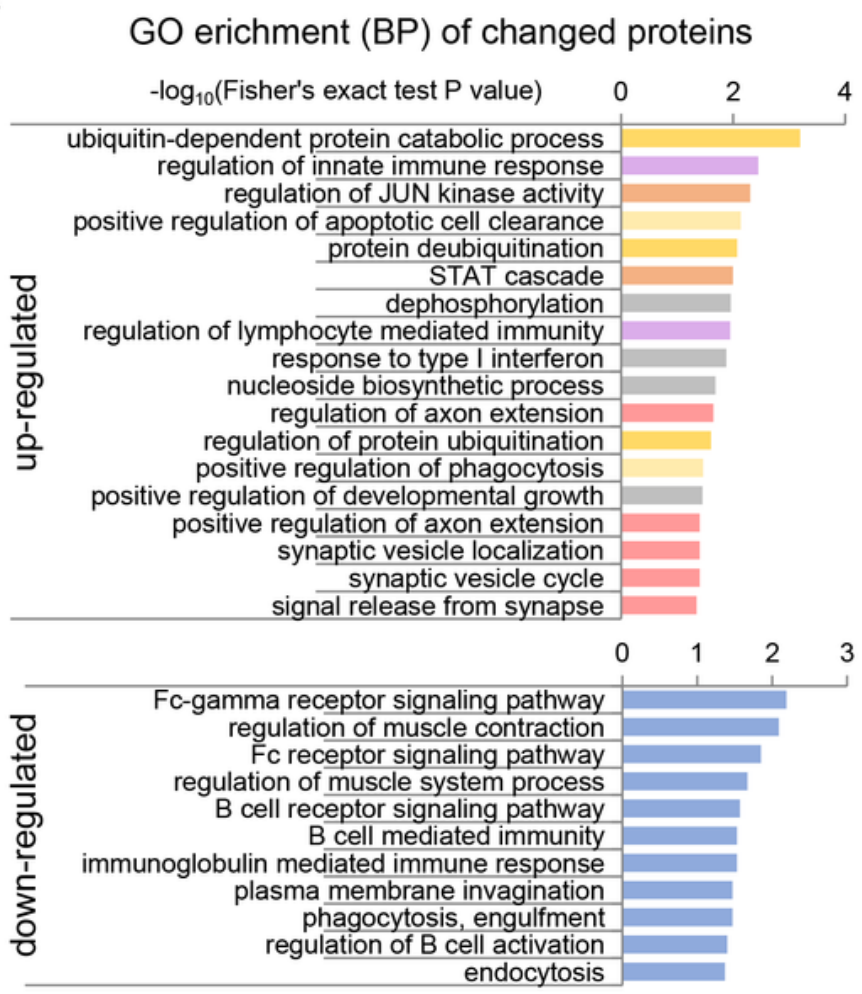

C
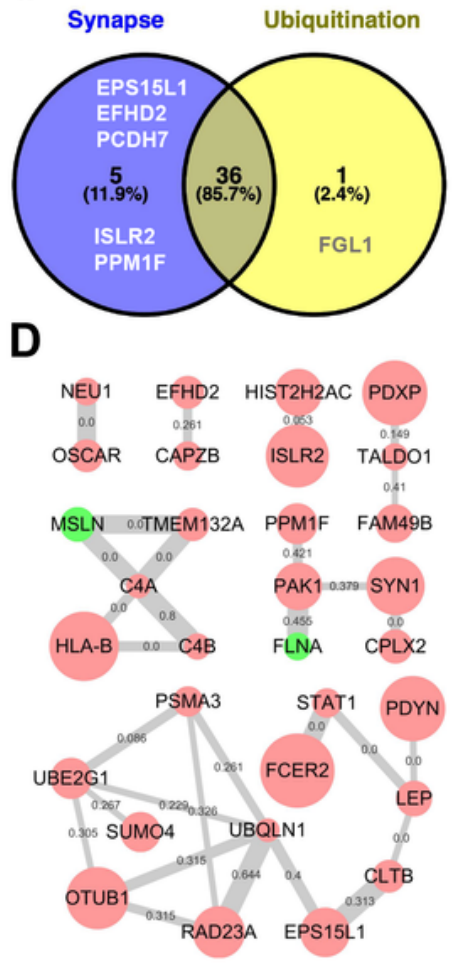

B

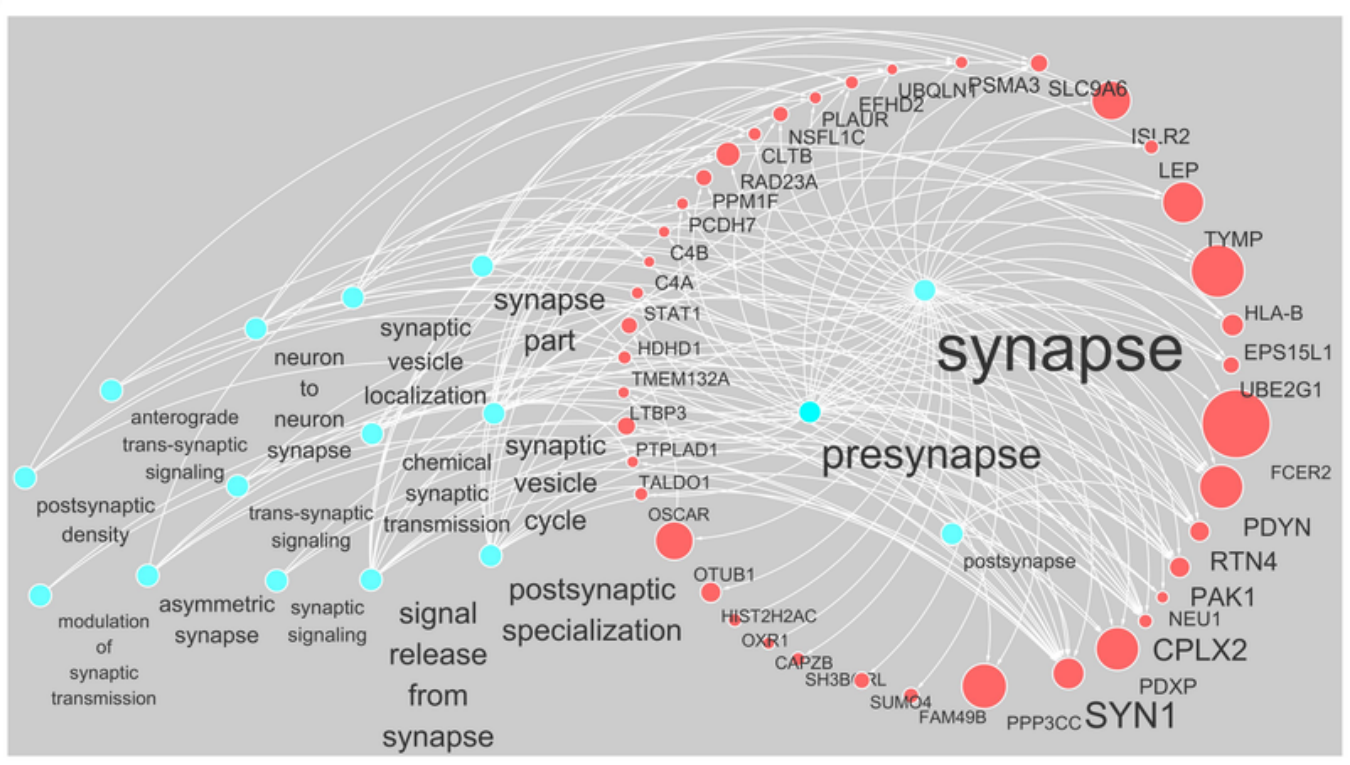

\section{Figure 4}

Proteins involved in synapse, ubiquitination, apoptosis, and innate and lymphocytes mediated immune were up-regulated in CSF samples of C-NORSE patients. (A) GO biological processes enrichment of up(upper panel) and down (lower panel)-regulated proteins of C-NORSE. (B) 41 of 42 up-regulated proteins related to synaptic $\mathrm{GO}$ terms. Red bubbles represent up-regulated proteins. Blue bubbles show GO-related terms. The size of red bubble indicates fold-change. The font size represents relative number of links. (C) 
Venny analysis of synaptic-related proteins and ubiquitination-involved proteins. 36 of 41 increased synaptic proteins were also related to ubiquitination. (D) PPI network of differentially expressed proteins. The interactions were acquired from STRING database and reconstructed by Cytoscape software. Red bubbles indicate up-regulated proteins. Green bubbles represent down-regulated proteins. The bubble size denotes relative fold-change. The link weight represents combined score and the number on the line shows the confidence of an experimentally determined interaction. BP, biological processes; GO, gene ontology; PPI, protein-protein interaction.

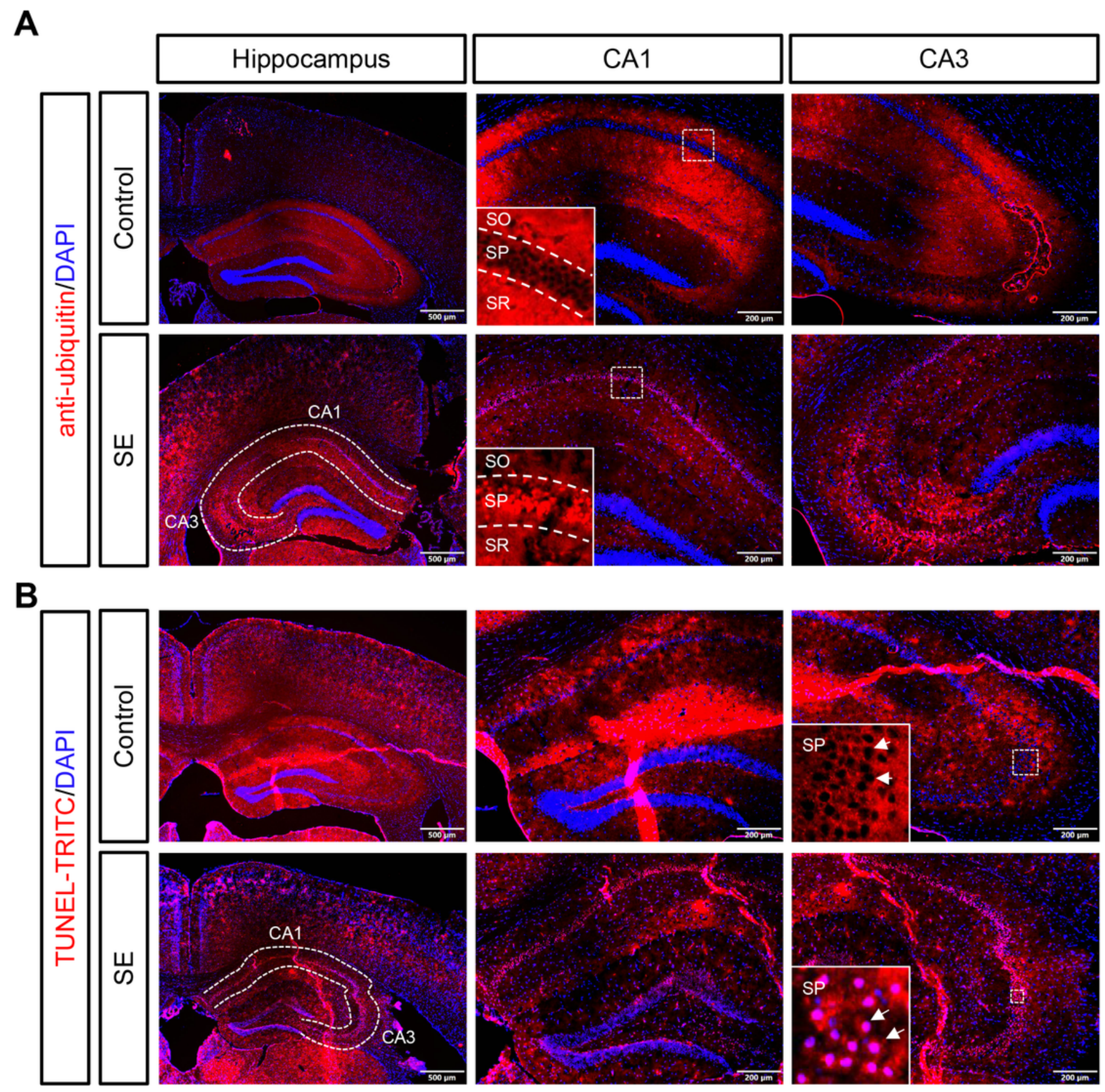

Figure 5 
Cell ubiquitination and apoptosis were enhanced in hippocampus of mouse with SE. (A) Immunostaining of ubiquitin in brain of a mouse with SE. Obviously increased ubiquitination signals (red) were observed in stratum pyramidale of hippocampal CA1 to CA3 regions of brain of a mouse with SE. Normal mouse was used as control. (B) TUNEL assay for apoptosis detection of brain of a mouse with SE. Significantly enhanced signals (TRITC red) were observed in hippocampal CA1 to CA3 regions of brain of a mouse with SE. Normal mouse was used as control. SE, status epilepticus; blue, nucleus stained with DAPI; SO, stratum oriens; SP, stratum pyramidale; SR, stratum radiatum; scale bars (in hippocampus) $=500 \mu \mathrm{m}$; scale bars (in CA1 and CA3) $=200 \mu \mathrm{m}$.

\section{anti-NMDAR E. NORSE}

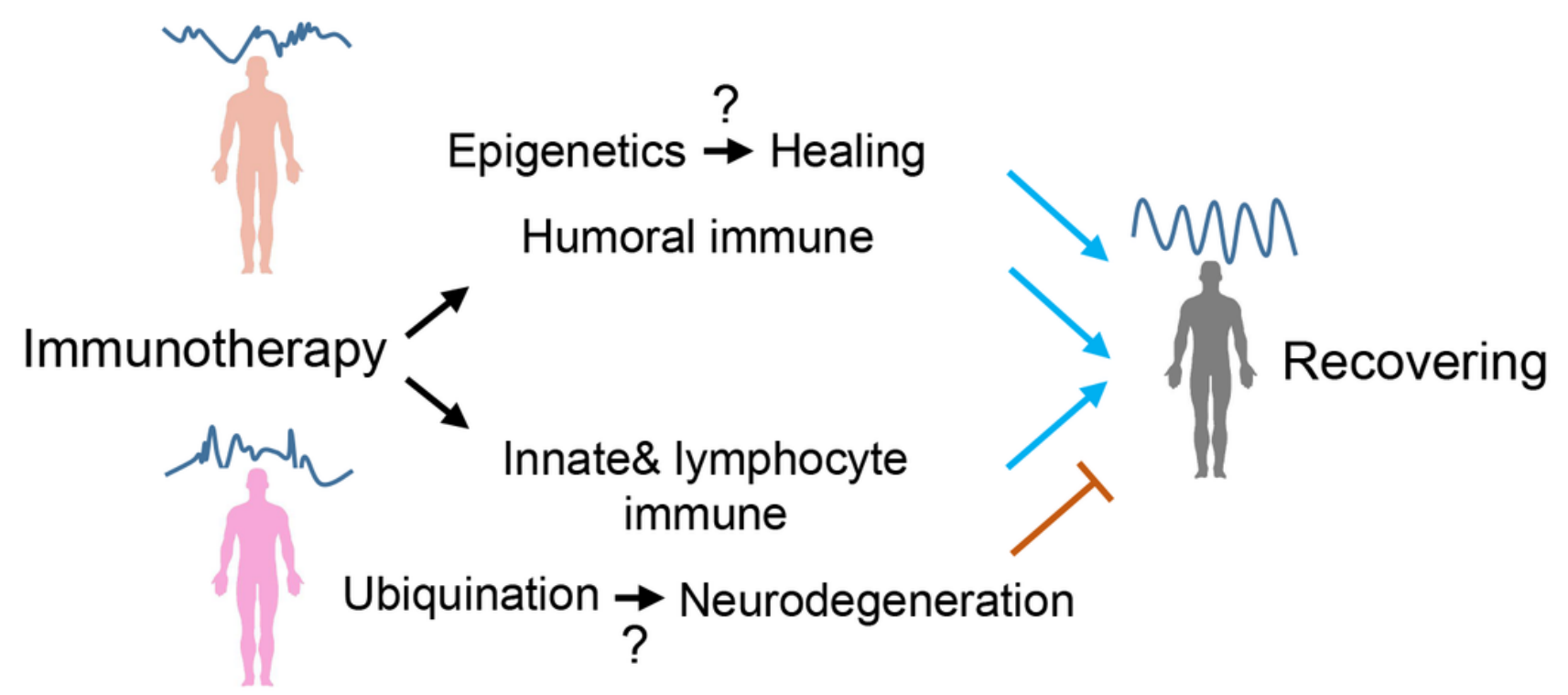

C-NORSE

Figure 6

A hypothesis model of anti-NMDAR encephalitis NORSE and C-NORSE. Anti-NMDAR encephalitis NORSE and cryptogenic-NORSE are two types of SE. According to the findings of this study, proteomes changed differently in these two diseases and the up-regulated proteins were closely correlated to disease severity. In anti-NMDAR encephalitis NORSE patients, humoral immune response was up-regulated, while innate and lymphocyte meidated immune response was up-regulated in C-NORSE patients, suggesting that these two diseases might respond to immunotherapy with different mechanisms. Wound healing was enhanced in anti-NMDAR encephalitis NORSE patients. Epigenetic regulation of gene expression might contribute to the regulation wound healing-associated pathways. In C-NORSE patients, synaptic proteins were up-regulated, as well as ubiquitination and cell apoptosis, which could disrupt synaptic homeostasis 
and cause SE. It probably plays a negative role in the treatment of disease. Appropriate immunotherapy approaches should be considered for treatment.

\section{Supplementary Files}

This is a list of supplementary files associated with this preprint. Click to download.

- Additionalfile1.xlsx

- Additionalfile2.xlsx

- Additionalfile3.xlsx

- Additionalfile4.xlsx

- Additionalfile5.xlsx

- Additionalfile6.xlsx

- Additionalfile7.xIsx

- s1.png

- s2.png

- s3.png

- s4.png

- s5.png

- s6.png

- s7.png

- s8.png

- s9.png

- s10.png

- s11.png

- s12.png

- s13.png

- s14.png

- s15.png 